\title{
Developmental Profiles of the Intrinsic Properties and Synaptic Function of Auditory Neurons in Preterm and Term Baboon Neonates
}

\author{
Sei Eun Kim, ${ }^{1}$ Seul Yi Lee, ${ }^{1}$ Cynthia L. Blanco, ${ }^{3}$ and $\odot$ Jun Hee Kim ${ }^{1,2}$ \\ ${ }^{1}$ Department of Physiology, ${ }^{2}$ Center for Biomedical Neuroscience, and ${ }^{3}$ Department of Pediatrics, University of Texas Health Science Center, San Antonio, \\ Texas 78229
}

\begin{abstract}
The human fetus starts to hear and undergoes major developmental changes in the auditory system during the third trimester of pregnancy. Although there are significant data regarding development of the auditory system in rodents, changes in intrinsic properties and synaptic function of auditory neurons in developing primate brain at hearing onset are poorly understood. We performed whole-cell patch-clamp recordings of principal neurons in the medial nucleus of trapezoid body (MNTB) in preterm and term baboon brainstem slices to study the structural and functional maturation of auditory synapses. Each MNTB principal neuron received an excitatory input from a single calyx of Held terminal, and this one-to-one pattern of innervation was already formed in preterm baboons delivered at $67 \%$ of normal gestation. There was no difference in frequency or amplitude of spontaneous excitatory postsynaptic synaptic currents between preterm and term MNTB neurons. In contrast, the frequency of spontaneous $\mathrm{GABA}_{\mathrm{A}}$ /glycine receptor-mediated inhibitory postsynaptic synaptic currents, which were prevalent in preterm MNTB neurons, was significantly reduced in term MNTB neurons. Preterm MNTB neurons had a higher input resistance than term neurons and fired in bursts, whereas term MNTB neurons fired a single action potential in response to suprathreshold current injection. The maturation of intrinsic properties and dominance of excitatory inputs in the primate MNTB allow it to take on its mature role as a fast and reliable relay synapse.
\end{abstract}

Key words: auditory brainstem; development; nonhuman primate; synaptic transmission; calyx synapse; MNTB

\section{Introduction}

In early brain development, auditory impairment can profoundly affect reading and speaking ability and learning and memory, all of which are associated with auditory processing disorders (Downie et al., 2002; Iliadou et al., 2008). Processing of auditory information begins in the brainstem, where specialized nuclei decode information regarding the onset and offset of sounds and their spatial origin (Wible et al., 2005; Grothe et al., 2010). Function and development of the auditory brainstem have mostly been studied in species that do not hear at birth, such as rat, mouse, and cat. In these species, hearing onset occurs 10-12 d after birth (Cant, 1998; Sonntag et al., 2011). In contrast, in humans hearing starts in utero. In particular, the third trimester ( $>65 \%$ of gestational age [GA]) is a critical time period for growth and maturation of the auditory system (Moore and Jef-

\footnotetext{
Received Nov. 7, 2013; revised July 11, 2014; accepted July 14, 2014

Author contributions: J.H.K. designed research; S.E.K., S.Y.L., and J.H.K. performed research; C.L.B. contributed unpublished reagents/analytic tools; S.E.K., S.Y.L., and J.H.K. analyzed data; J.H.K. wrote the paper.

We thank Drs. Christopher Kushmerick, Emmanuelle Barret, René Rentría, Robert Renden and John Johnson for valuable discussion and comments, and also appreciate Michelle Leland, DVM for technical assistance. This work was supported by the March of Dimes Basil O'Connor Starter Research Award (FY11-570) and NIDCD R01 (R01DC013157) to J.H.K.

The authors declare no competing financial interests.

Correspondence should be addressed to Dr. Jun Hee Kim, Department of Physiology, University of Texas Health Science Center, 7703 Floyd Curl Drive, San Antonio, TX 78229. E-mail: kimjh@uthscsa.edu.

DOI:10.1523/JNEUROSCI.4734-13.2014

Copyright $\odot 2014$ the authors $\quad 0270-6474 / 14 / 3411399-06 \$ 15.00 / 0$
}

fery, 1994; Hall, 2000). Developmental changes in structures coincide with behavioral changes as the human fetus can hear and react to auditory signals at $\sim 65 \%$ GA (Querleu et al., 1989; Moore and Linthicum, 2007). However, little is known about structural and functional changes to synapses during early development in the primate auditory brainstem that are important for achieving speed, temporal precision, and reliability of sound transmission required of auditory circuits.

The auditory brainstem responses (ABRs) from preterm and term baboons indicate that hearing onset takes place $~ 70 \% \mathrm{GA}$ (Edwards et al., 1994). The patterns of development of both ABR waveform morphology and latency were consistent with those seen in the human neonate, with a rapid maturation of the response during the perinatal period, followed by a slower development to adult values (Vles et al., 1987; Edwards et al., 1994). However, to interpret changes in the ABRs in terms of the underlying synaptic function requires electrophysiological recordings at the single synapse level from the developing primate brain. Here, we studied the intrinsic excitability and synaptic activities of the medial nucleus of trapezoid body (MNTB) neurons in the superior olivary complex (SOC) from preterm and term baboon neonates. The SOC acts a pivotal sign inverter in brainstem sound localization.

\section{Materials and Methods}

Animals. A total of seven baboon brains, including three term and four preterm baboons of either sex, were obtained from the laboratory of Dr. Cynthia L Blanco, Department of Pediatrics. Animals were used in ac- 
cordance with approved University of Texas Health Science Center, San Antonio Institutional Animal Care and Use Committee protocols. Preterm baboons were delivered at $67 \%$ of gestation at the Texas Biomedical Research Institute in San Antonio. Preterm baboon care was recently described in detail (Blanco et al., 2013). Briefly, term baboons were naturally delivered (at $185 \mathrm{~d}$ GA), whereas preterm baboons were delivered via cesarean section at $125 \pm 2 \mathrm{~d}$ GA ( $67 \%$ of GA) and were intubated immediately after birth and chronically ventilated for a planned survival of $14 \mathrm{~d}$ in a neonatal intensive care unit. Central intravenous lines were placed shortly after birth for fluid management and parenteral nutrition. Preterm baboons were treated with corticosteroids, antibiotics, ketamine, valium, vitamin $\mathrm{K}$, and blood transfusions (Blanco et al., 2013).

Slice preparation. Brainstem slices were prepared from extracted full-term and preterm baboon brainstem after necropsy. The brainstem was immersed in ice-cold low-calcium aCSF containing the following (in $\mathrm{mm}$ ): 125 $\mathrm{NaCl}, 2.5 \mathrm{KCl}, 3 \mathrm{MgCl}_{2}, 0.1 \mathrm{CaCl}_{2}, 25$ glucose, $25 \mathrm{NaHCO}_{3}, 1.25 \mathrm{NaH}_{2} \mathrm{PO}_{4}, 0.4$ ascorbic acid, 3 myoinositol, and 2 Na-pyruvate, bubbled with carbogen $\left(95 \% \mathrm{O}_{2}, 5 \% \mathrm{CO}_{2}\right.$; $\mathrm{pH}$ 7.3-7.4; osmolality of 310-320 mOsm). Transverse brainstem slices (200 $\mu \mathrm{m}$ thick) were prepared using a microtome (VT1200S, Leica). After cutting, the slices were transferred to an incubation chamber containing normal aCSF bubbled with carbogen and maintained at $35^{\circ} \mathrm{C}$ for $30 \mathrm{~min}$ and thereafter at room temperature. Normal aCSF was the same as the slicing aCSF, but with $1 \mathrm{~mm} \mathrm{MgCl}_{2}$ and $2 \mathrm{~mm} \mathrm{CaCl}_{2}$.

Electrophysiology. Whole-cell patch-clamp recordings were performed in normal aCSF at room temperature $\left(22^{\circ} \mathrm{C}-24^{\circ} \mathrm{C}\right)$. Pipette solution contained the following (in $\mathrm{mm}$ ): 130 K-gluconate, $20 \mathrm{KCl}, 5 \mathrm{Na}_{2}$-phosphocreatine, 10 HEPES, 5 EGTA, $4 \mathrm{Mg}$-ATP, and 0.3 GTP, $\mathrm{pH}$ adjusted to 7.3 with $\mathrm{KOH}$. EPSCs or action potentials (APs) were recorded in normal aCSF using the voltage or current-clamp mode of the EPC-10 (HEKA Electronik). Patch electrodes had resistances of 2.5-3 $\mathrm{M} \Omega$, and the initial uncompensated series resistance (Rs) was $<20 \mathrm{M} \Omega$. Recordings were not corrected for the predicted liquid junction potential of $11 \mathrm{mV}$. APs were elicited by current injection in current-clamp mode or with a bipolar platinum/iridium electrode placed near the midline spanning the afferent fiber tract of the MNTB. Postsynaptic currents were measured under whole-cell voltage-clamp with a holding potential of $-70 \mathrm{mV}$. Data were filtered at $2.9 \mathrm{kHz}$ and digitized at a $10-50 \mu$ s sampling intervals.

Immunohistochemistry. After electrophysiological recordings, brainstem slices $(150-200 \mu \mathrm{m})$ were fixed with $4 \%$ (w/v) PFA in phosphate buffer solution (PBS) for $30 \mathrm{~min}$. Free-floating sections were blocked in $4 \%$ goat serum and $0.3 \%$ Triton X-100 in PBS for $1 \mathrm{~h}$. Primary antibodies were guinea pig polyclonal anti-vesicular glutamate transporter 1 (VGluT1, 1:3000, Millipore Bioscience Research Reagents), antimicrotubule associated protein 2 (MAP2, 1:200, Millipore). Staining was reported by incubation with different Alexa dye-conjugated secondary antibodies (1:500, Invitrogen) for $2 \mathrm{~h}$ at room temperature. Stained slices were viewed using a $40 \times$ oil-immersion objective on a confocal laser scanning microscope (LSM-510, Zeiss).

Quantification. Cell density was determined by counting the number of cell bodies visible on the surface of the slice within an area of (100 $\mu \mathrm{m}^{2}$ ). This area is significantly smaller than the area occupied by the
MNTB in all slices analyzed, thus avoiding differences due to cutting angle or rostral/caudal location. GABAergic inputs to the MNTB were quantified by counting the number of VGAT puncta on the cell body. The pattern of GABAergic input to the medial superior olive (MSO) was quantified by measuring the distance of VGAT puncta to the axis defined by the line of MSO neurons (Kapfer et al., 2002).

Analysis and statistics. Electrophysiological data were analyzed and presented using Igor Pro (Wavemetrics). Postsynaptic currents were detected and analyzed with Mini Analysis (Synaptosoft). Histograms of postsynaptic current amplitude were fit with the sum of Gaussian distributions. Comparisons between mean values were statistically analyzed using the Mann-Whitney $U$ test for two independent samples. Differences were deemed statistically significant when $p$ values were $<0.05$.

\section{Results}

Morphological changes in the SOC nuclei of developing baboon brains

The MNTB was identified as the most medial nucleus along the auditory fibers after crossing the midline of the brainstem. The MNTB started at $\sim 0.7 \mathrm{~mm}$ from midline in preterm and at $\sim 2$ $\mathrm{mm}$ in term baboon. During development, the area occupied by the MNTB on the surface of the slice more than doubled from 
A

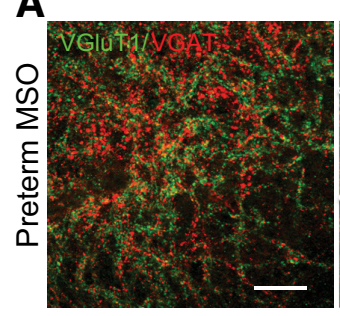

B

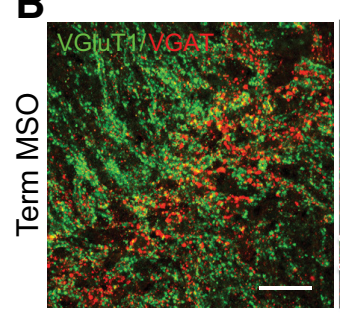

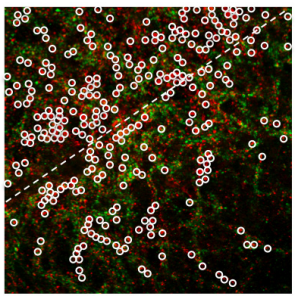

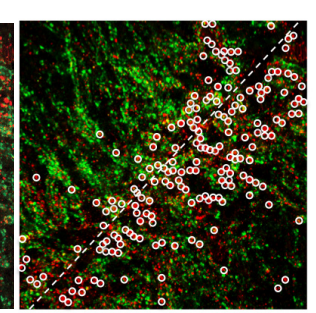

Preterm MSO

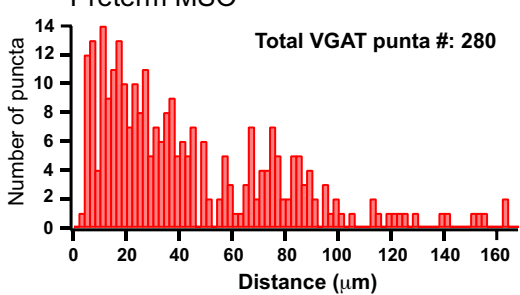

Term MSO

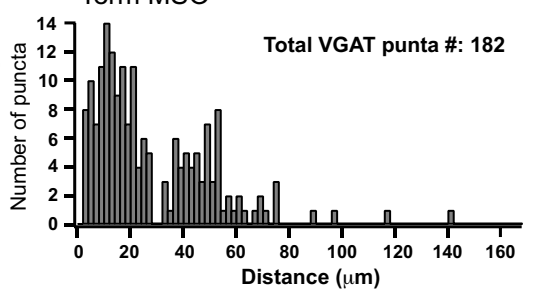

C

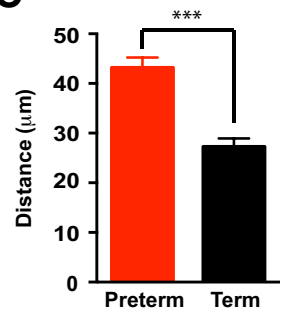

Figure 2. The spatial pattern of inhibitory inputs to the MSO in preterm and term baboon neonates. $\boldsymbol{A}, \boldsymbol{B}, \mathrm{VGAT}$ (red) and VGluT1 (green) staining in the MSO in preterm ( $\boldsymbol{A}$ ) and term baboon brainstems $(\boldsymbol{B})$. Scale bar, $50 \mu \mathrm{m}$. VGAT puncta were marked with the circle (area: 430 pixels, mean signal intensity $>70$, white circles) in the preterm and term MSO. The broken line indicates the layer of MSO soma for measuring the distance between the MSO soma and VGAT puncta. In preterm MSO, VGAT puncta spread out from MSO soma, whereas VGAT puncta oriented to the layer of MSO soma in term MSO. Inset, Histogram of VGAT puncta distribution. C, Mean distance of VGAT puncta from the layer of MSO soma in preterm and term MSO. ${ }^{* * *} p<0.001$.

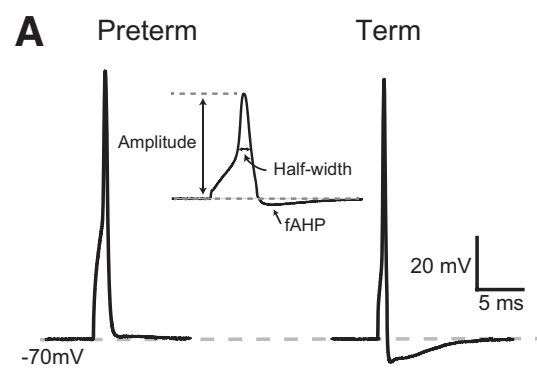

B Amplitude

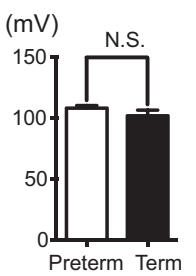

D fAHP
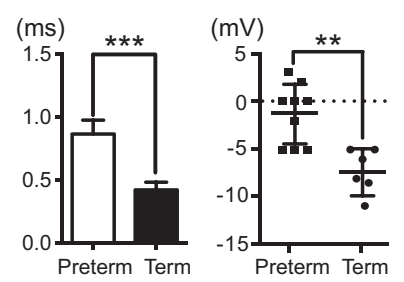

E

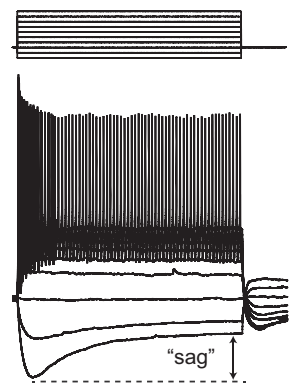

Term
F Input Resistance

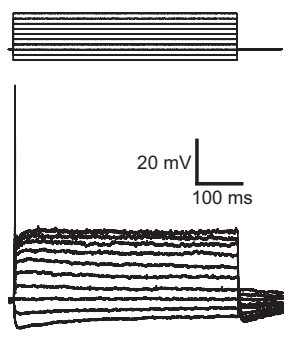

Figure 3. The MNTB neurons from preterm baboon are hyperexcitable. A, APs from MNTB neurons were evoked by a brief current injection ( $500 \mathrm{pA} \mathrm{for} 2 \mathrm{~ms}$ ) in whole-cell current-clamp mode. Inset, Example of a single AP for showing how to measure the amplitude, half-width, and fAHP. $\boldsymbol{B}-\boldsymbol{D}$, Summary of AP properties, including amplitude $(\boldsymbol{B})$ and half-width $(\boldsymbol{C})$, and amplitude of fAHP (D) in preterm and term MNTB neurons. $\boldsymbol{E}$, APs of MNTB neurons in response to long current injections (steps from $-100 \mathrm{pA}$ to $350 \mathrm{pA}, 500 \mathrm{~ms}$ ). Inset, "Sag" potential was measured by subtracting the steady-state potential from the peak potential during a hyperpolarizing current injection. $\boldsymbol{F}, \boldsymbol{G}$, Summary of input resistance $(\boldsymbol{F})$ and the amplitude of depolarizing-sag from the peak hyperpolarization of $-82 \pm 2.3 \mathrm{mV}$ (injected with $-50 \mathrm{pA}$ for preterm and $-100 \mathrm{pA}$ for term neurons) to the steady state $(\boldsymbol{G})$. ${ }^{*} p<0.05,{ }^{* *} p<0.01,{ }^{* * *} p<0.001$. N.S., Not significant.

$92,000 \pm 30,400 \mu \mathrm{m}^{2}$ to $196,000 \pm 24,900 \mu \mathrm{m}^{2}$, whereas the cell density fell from $14.2 \pm 1.2$ per $\left(100 \mu \mathrm{m}^{2}\right)$ in preterm MNTB to $6 \pm 0.8 \operatorname{per}\left(100 \mu \mathrm{m}^{2}\right)$ in term MNTB $(n=6$ and $n=6$ slices, $p=$ 0.002 ; Fig. $1 A, B)$. This result is comparable with that in the human auditory system in which rapid growth of the brainstem occurs during the perinatal period (Moore and Linthicum, 2007). During this period, the MSO, a dorsoventrally orientated column

G Sag-depolarization

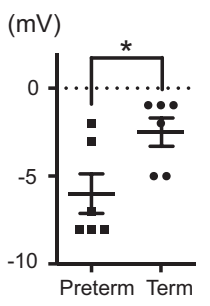

of neurons, located laterally to the MNTB becomes longer and narrower (Fig. $1 A, B)$. Double labeling with MAP2 and VGluT1 indicates glutamatergic inputs onto most MNTB and MSO neurons in term baboon brainstem (Fig. 1C). We filled MNTB neurons with Alexa-568 dye during whole-cell recordings and then fixed and stained the slices for VGluT1 (Fig. 1D,E). MNTB neurons present oval soma with diameters of $18.3 \pm 0.9 \mu \mathrm{m}$ and $18.9 \pm 1.5 \mu \mathrm{m}$ in preterm and term MNTB neurons $(n=20$ and $n=6, p=$ 0.74). Staining for VGluT1 revealed the tight axosomatic synapse formed by the calyx terminal onto the MNTB neuron with thick finger-like processes (Fig. $1 D, E)$. There was no significant difference in the shape or size of the calyx terminals between in term and preterm brainstems. However, staining for the vesicular GABA transporter (VGAT) revealed a reduction in number of VGAT puncta from $18.4 \pm 1.25$ puncta/cell surface in preterm MNTB neurons to $11.3 \pm$ 0.47 puncta/cell surface in term MNTB neurons ( $n=7$ and $n=4, p=0.006$; Fig. $1 F, G)$.

In the preterm baboon brainstem, the MSO displayed strong labeling for VGAT (red) and weak labeling for VGluT1 (green), whereas in the term baboon the MSO exhibited strong VGluT1 labeling on dendrites (Fig. 2A,B). We also found a different pattern of VGAT distribution in preterm and term baboons. In preterm MSO, intense staining for VGAT was visible on both cell bodies and dendrites, whereas in term MSO VGAT puncta are concentrated on the column of cell bodies. The mean distance from the soma of MSO neuron to VGAT puncta was $43.2 \pm 2.1 \mu \mathrm{m}(n=$ 
280 puncta) in preterm and $27.3 \pm 1.7$ $\mu \mathrm{m}$ in term MSO $(n=182$ puncta, $p<$ 0.0001; Fig. 2C). Thus, similar to other species with low-frequency hearing (Kapfer et al., 2002), significant changes in the degree of innervation and the spatial pattern of inhibitory inputs to the MSO occur during the period after hearing onset in the baboon.

\section{Developmental decrease in MNTB excitability in baboon brainstem} The resting membrane potentials of MNTB neurons were $-71 \pm 0.9 \mathrm{mV}$ in preterm and $-70 \pm 0.6 \mathrm{mV}$ in term baboons ( $n=8$ and $n=8$, respectively, $p=$ 0.29 ). Minimal currents necessary to elicit an AP were $145 \pm 24.6 \mathrm{pA}$ in preterm and $180 \pm 35.9 \mathrm{pA}$ in term $(p=0.70)$. AP thresholds, determined by the inflection point, were $-44 \pm 1.1 \mathrm{mV}$ in preterm and $-48 \pm 2.6 \mathrm{mV}$ in term MNTB neurons ( $n=8$ and $n=8, p=0.31$ ). Short depolarizing current injection (500 pA, 2 ms) evoked a single AP with amplitude of $107 \pm 2.1 \mathrm{mV}$ and $101 \pm 4.6 \mathrm{mV}$ (from the resting potential to the peak of AP) in preterm and term MNTB neurons $(n=8$ and $n=8, p=0.20$; Fig. $3 A, B$ ). Although amplitude and threshold of APs were similar, AP half-width decreased by $50 \%$ during development from $0.86 \pm 0.11 \mathrm{~ms}$ in preterm to $0.42 \pm 0.06 \mathrm{~ms}$ in term MNTB neurons ( $n=8$ and $n=8, p=0.0009$; Fig. $3 C)$. In addition, term MNTB neurons showed a significantly larger fast afterhyperpolarization (fAHP) than preterm MNTB neurons (amplitude of fAHP: $-7.3 \pm 0.98 \mathrm{mV}$ and $-1.3 \pm 1.02 \mathrm{mV}$, $n=7$ and $n=8, p=0.0032$; Fig. $3 D$ ).

Most term MNTB neurons (6 of 8 recordings) exhibited a phasic firing pattern with only a single AP in response to a long suprathreshold current injection (200 pA, $500 \mathrm{~ms})$. In contrast, most preterm MNTB neurons (5 of 8 recordings) displayed a tonic firing pattern with a burst of APs $(80 \pm 10.8 \mathrm{~Hz}, n=5)$ in response to the suprathreshold depolarizing current injections (200 pA; Fig. 3E). Possible reasons for higher excitability in preterm $\mathrm{MNTB}$ neurons include higher input resistance of $(163 \pm 11.2 \mathrm{M} \Omega$ in preterm vs $104 \pm$ $13.6 \mathrm{M} \Omega$ in term MNTB, $n=8$ and $n=6, p=0.006$; Fig. $3 F$ ). During injection of hyperpolarizing current, preterm MNTB neurons displayed a prominent depolarizing-sag, suggesting that immature MNTB neurons express a nonselective hyperpolarization-activated cation current $\left(\mathrm{I}_{\mathrm{h}}\right)$ (Banks et al., 1993; Koch et al., 2004) (Fig. 3E). To compensate for the difference in input resistance, we compared the size of depolarizing-sag at the peak hyperpolarization of $-82 \pm 2.3 \mathrm{mV}$ in preterm neurons injected with $-50 \mathrm{pA}$ and in term neurons injected with $-100 \mathrm{pA}$. We observed a significantly larger depolarizing-sag in

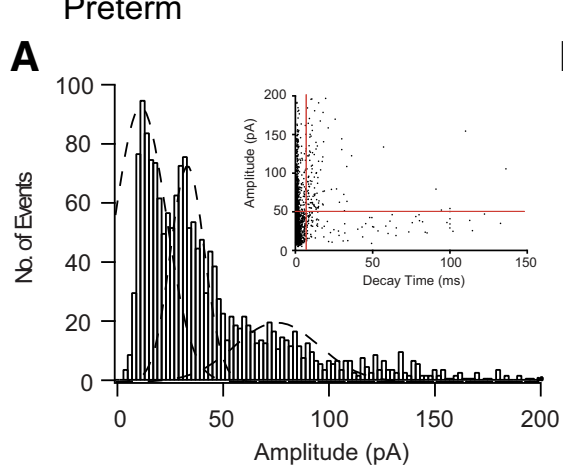

B
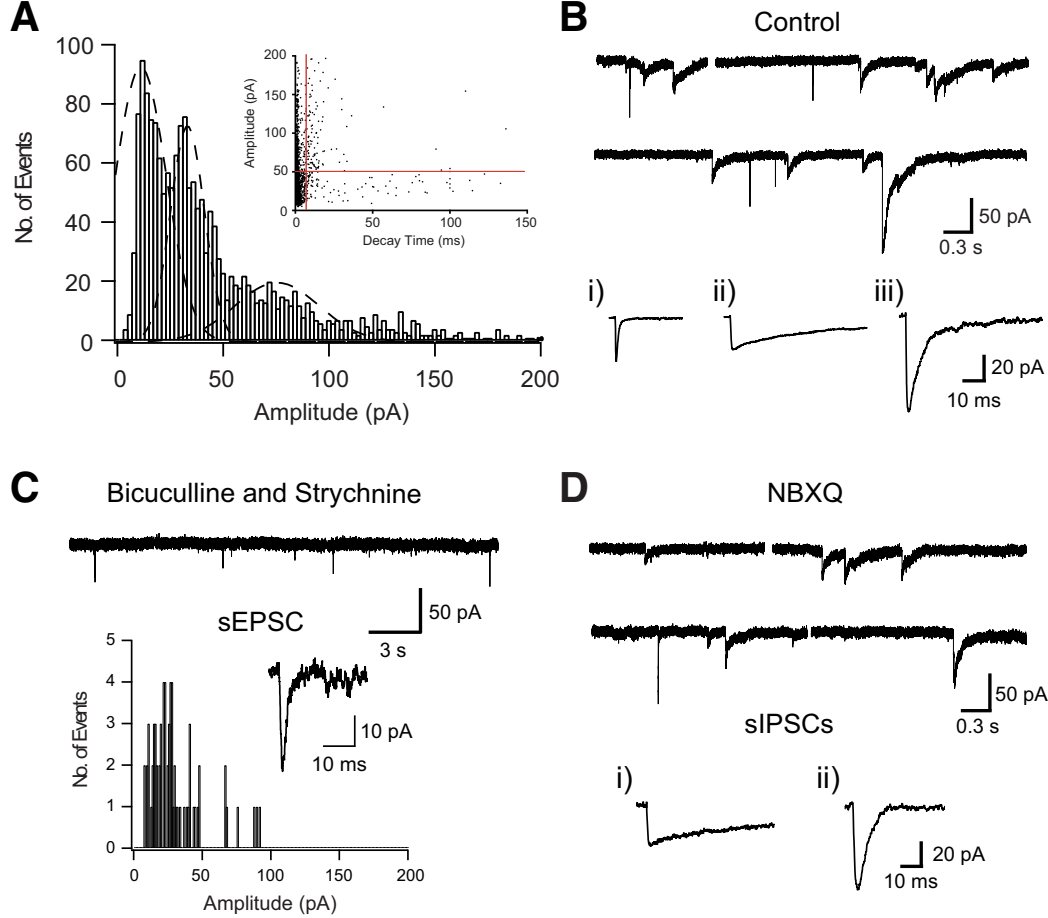

D

NBXQ
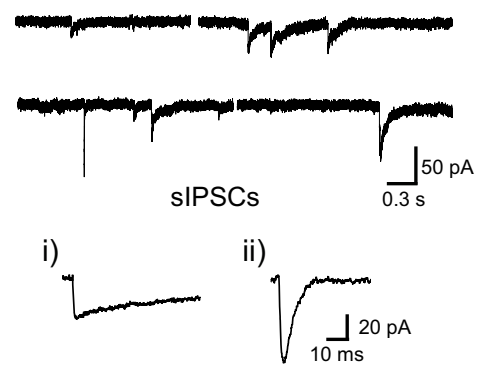
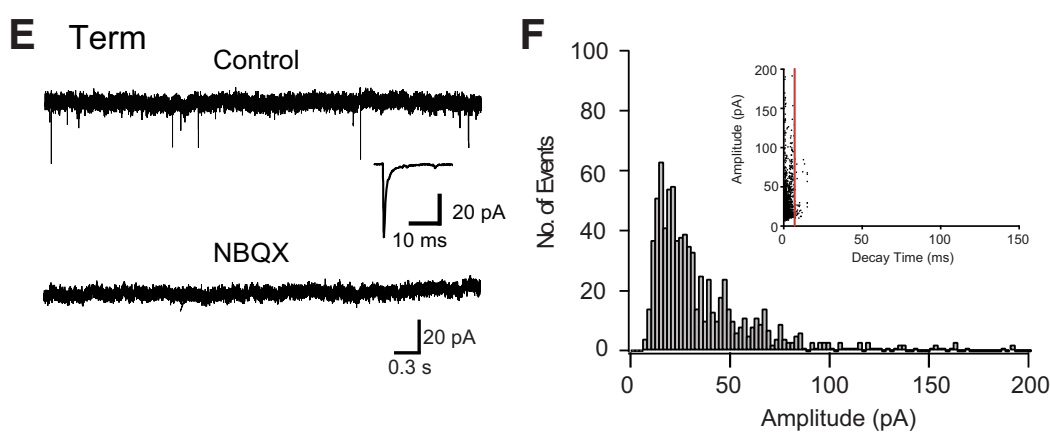

Figure 4. Spontaneous PSC recordings from a single principal neuron in the MNTB in term and preterm baboon. $\boldsymbol{A}$, Histogram of amplitude distribution of spontaneous PSCs in control from preterm MNTB neurons. Gaussian fits to histograms of PSC amplitudes pooled from 13 neurons showed three peaks. Inset, Distribution of amplitude plotted versus decay time of spontaneous PSCS. Red lines indicate $7 \mathrm{~ms}$ (vertical line) and $50 \mathrm{pA}$ (horizontal line). $\boldsymbol{B}$, Representative traces of PSCs recorded in preterm MNTB Biii, Average trace of PSCs in three different groups in control with the same scale for amplitude and time. $\boldsymbol{C}_{\boldsymbol{C}}$ Representative traces of spontaneous EPSCs recorded in the presence of bicuculline (50 $\mu \mathrm{m}$ ) and strychnine (1 $\mu \mathrm{M}, \boldsymbol{C})$ in preterm MNTB neuron. Inset, Histogram of amplitude distribution of spontaneous EPSCs and representative event of spontaneous EPSC. $\boldsymbol{D}$ s IPSCs in the presence of NBQX $(100 \mu \mathrm{m}, \boldsymbol{D})$. Inset, Average trace of sIPSCs (Di, Dii) in preterm MNTB neurons. $\boldsymbol{E}_{\text {, }}$ Representative traces of spontaneous PSCs recorded from the term MNTB neuron in control and in the presence of NBQX. Inset, Average trace of PSCs in term MNTB neurons. F, Histogram of amplitude distribution of spontaneous PSCs in control. Inset, Plot of amplitude against decay time of spontaneous PSCs. Red vertical line indicates $7 \mathrm{~ms}$.

preterm neurons $(6 \pm 1.1 \mathrm{mV}$ in preterm and $2.5 \pm 0.8 \mathrm{mV}$ in term MNTB neurons, $n=7$ and $n=7, p=0.026$, Fig. $3 G$ ).

Spontaneous synaptic activities in the MNTB are altered during late fetal development in baboon brainstem

MNTB principal neurons received glutamatergic inputs from calyx nerve terminals and glycinergic/GABAergic inputs exhibiting spontaneous postsynaptic currents (PSCs) in preterm and term brainstem (Fig. $4 A, B$ ). In preterm MNTB, spontaneous PSCs were heterogeneous with respect to their pharmacological and kinetic properties. Gaussian fits to histograms of PSC amplitudes pooled from 13 neurons showed three peaks (Fig. 4A). Based on the distribution of amplitude and decay time, we identified three 
A sEPSPs

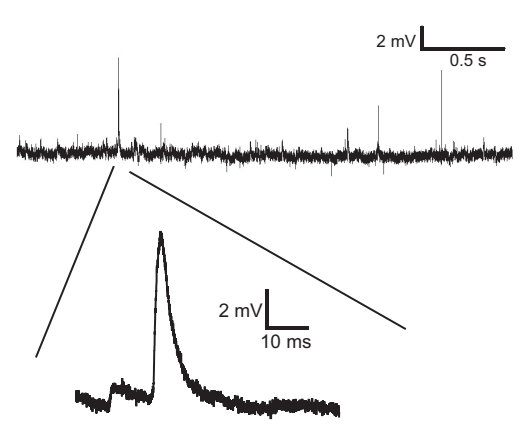

B

eEPSC

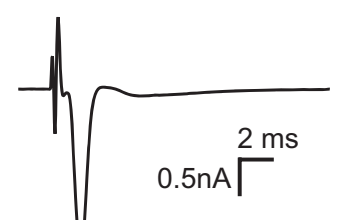

C

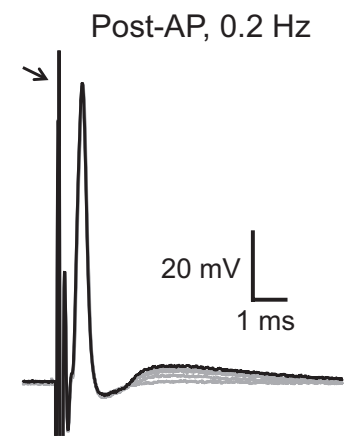

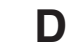

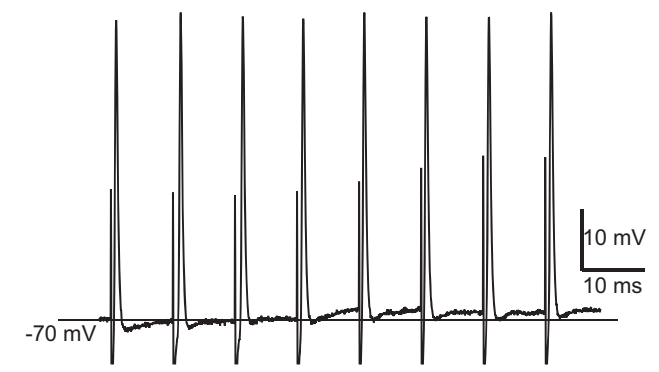

Figure 5. Spontaneous EPSPs, evoked EPSCS, and postsynaptic APs in MNTB neurons. $A$, Spontaneous EPSPs were recorded in current-clamp recordings of MNTB neurons at resting membrane potential $(-70 \mathrm{mV})$. Inset, A single EPSP in expanded time scale. B, Afferent fiber stimulation (at $0.2 \mathrm{~Hz}$ ) on auditory axon fibers at the midline evoked EPSC in voltage-clamp recordings. $C$, Superimposed postsynaptic APs (at $0.2 \mathrm{~Hz}, 5 \mathrm{APs}$ ) in the MNTB neuron under current-clamp recordings. Arrow indicates a stimulus artifact. $\boldsymbol{D}$, Postsynaptic AP train evoked by afferent fiber stimulation at $100 \mathrm{~Hz}$.

different groups of PSCs in preterm MNTB neurons. The first group of PSCs had amplitudes of $-41 \pm 3.2 \mathrm{pA}$ and a fast decay time constant of $1.7 \pm 0.2 \mathrm{~ms}$ (Fig. $4 \mathrm{Bi}$ ). The second group of PSCs had amplitudes of $-22 \pm 1.0 \mathrm{pA}$ and much slower decay time constant of $-71 \pm 8.2 \mathrm{~ms}$ (Fig. 4Bii). The third group of PSCs showed much larger amplitudes of $-82 \pm 4.0 \mathrm{pA}$ and intermediate decay time constants with a mean of $9.8 \pm 1.5 \mathrm{~ms}$ (Fig. 4Biii). In the presence of bicuculline $(50 \mu \mathrm{M})$ and strychnine (1 $\mu \mathrm{M})$, blockers for $\mathrm{GABA}_{\mathrm{A}}$ and glycine receptors, respectively, remaining putative excitatory PSCs (EPSCs) had fast decay (Fig. $4 C)$. Conversely, in the presence of NBQX $(100 \mu \mathrm{M})$, an AMPA receptor antagonist, remaining putative inhibitory PSCs (IPSCs) corresponded to the intermediate and slow kinetic groups described above (Fig. 4Di,Dii). Consistent with the immunostaining for VGluT1 and VGAT, these data indicate that preterm baboon MNTB neurons are innervated by both glutamatergic and glycinergic/GABAergic inputs.

In contrast, term MNTB neurons displayed a single population of spontaneous PSCs with mean amplitude of $-40.02 \pm 5.2$ $\mathrm{pA}, 10-90 \%$ rise times of $0.73 \pm 0.04 \mathrm{~ms}$, and decay time constant $(\tau)$ of $1.35 \pm 0.13 \mathrm{~ms}$. The size and kinetics and median amplitude of PSCs recorded from term MNTB neurons were similar in size and kinetics to the first group of PSCs described above for preterm MNTB neurons (Fig. $4 E, F$ ). These spontaneous PSCs were completely blocked by NBQX (100 $\mu \mathrm{M})$, indicating that they were AMPA receptor-mediated EPSCs. Thus, despite the presence of labeling for VGAT in term MNTB, the majority of spontaneous PSCs appear to be AMPA receptormediated EPSCs.

In whole-cell current-clamp recordings from term MNTB neurons, spontaneous synaptic inputs were subthreshold and

MNTB neurons did not fire spontaneous APs (Fig. 5A). To examine whether synaptic input can evoke a postsynaptic AP, we evoked a presynaptic AP by afferent fiber stimulation at the brainstem midline. Evoked EPSCs with an amplitude of $3.0 \pm$ $0.27 \mathrm{nA}(n=3)$ evoked postsynaptic APs in term brainstem $(n=2$; Fig. $5 B)$. These evoked postsynaptic APs displayed a distinct fAHP followed by afterdepolarization (Fig. 5C). Superimposed APs evoked at $0.2 \mathrm{~Hz}$ showed essentially no timing jitter of spikes (AP latency; from stimulus artifact to the peak of AP, $0.7 \pm 0.15 \mu \mathrm{s}, n=2$ ), indicating high fidelity of synaptic transmission in term MNTB-calyx synapses. Term MNTB neurons can also follow $100 \mathrm{~Hz}$ stimulation without postsynaptic AP failure, and the range of AP latency shift was from $0.85 \mu$ s to $1.25 \mu$ s during AP train (between the first and 10th AP; Fig. 5D).

\section{Discussion}

Structural and functional maturation of auditory synapses during early development is critical for proper neural circuitry and for auditory processing in the developing brain. We characterized intrinsic properties and synaptic inputs of MNTB principal neurons in the auditory brainstem in term and preterm baboon brains. During perinatal development after hearing onset, MNTB neurons acquired a phasic firing pattern with a reduction in input resistance. At the same time, inhibitory inputs to the MNTB neuron declined and glutamatergic innervation became the dominant inputs. The transition of inhibitory inputs during the last trimester was not specific to the MNTB. The MSO also undergoes the refinement of inhibitory inputs, although inhibitory inputs to the MSO persisted in term baboon.

\section{Developmental transitions in inhibitory and excitatory inputs in the MNTB around hearing onset}

Previous immunohistochemical study in the SOC of adult rhesus monkey demonstrated that the MNTB soma was encircled by a dense assembly of synaptophysin-positive terminals, which corresponded to the calyces of Held (Bazwinsky et al., 2005). In both preterm and term baboon neonates, the calyx of Held terminal exhibited thick, finger-like processes, as observed in adult rhesus monkey. We observed no difference in the morphology of the calyx terminals or in the size or kinetics of spontaneous EPSCs recorded in MNTB neurons between preterm and term baboons (Figs. 1 and 4). This result suggests that glutamatergic innervation to the MNTB was morphologically and functionally well developed around hearing onset in preterm baboon. The major developmental change observed in the MNTB was a reduction of spontaneous inhibitory inputs. At the beginning of the third trimester, the maturation of glutamatergic synapses in the MNTB could be critical for achieving the proper transmission of auditory signals in the auditory pathway in the primate.

Rodent MNTB neurons are innervated by glutamatergic inputs at E17 (Hoffpauir et al., 2010), and at postnatal day 0 (P0) GABAergic inputs are major inhibitory inputs in parallel with 
glutamatergic inputs. After hearing onset ( $\sim 2$ weeks postnatal in rodents), a shift from GABAergic to glycinergic transmission is one of the major postnatal developmental changes in the MNTB (Turecek and Trussell, 2002; Awatramani et al., 2005). Our results demonstrate that spontaneous IPSCs in the MNTB are readily detectable at $67 \%$ gestation but are significantly reduced or undetectable in term MNTB neurons, although VGAT labeling of MNTB neurons is clearly present. Although we do not have evidence of glycinergic immunostaining in the MNTB in baboon neonates, one possible explanation is that spontaneous (asynchronous) GABA or glycine release may decrease during activitydependent refining of inhibitory inputs to the auditory brainstem after hearing onset. Synaptic inputs compete during formation of the calyx of Held synapse (Holcomb et al., 2013), and this decrease of inhibitory inputs may enhance the safety factor for excitatory neurotransmission at the MNTB.

\section{Changes in intrinsic properties and excitability of MNTB principal neurons during early development}

In term baboon, MNTB neurons exhibited phasic firing with a larger fAHP and a shorter AP half-width, whereas preterm MNTB neurons displayed a larger sag-depolarization and fired bursts of APs in response to constant depolarizing pulses (Fig. 3). These results were similar to those reported for immature rats during the first postnatal week (Hoffpauir et al., 2010; Rusu and Borst, 2011). Preterm MNTB neurons displayed a tonic firing pattern and a much higher input resistance $\left(\mathrm{R}_{\text {input }}\right)$. Age-related decreases in membrane resistance could be explained by an increase in cell membrane area (Hoffpauir et al., 2010). However, our immunostaining results showed no difference in the size of MNTB neurons or in the extent of dendritic development between preterm and term baboon brainstems (Fig. 1C,D). Therefore, it is more likely that decreased resistance is due to a developmental increase in the expression of ion channels. One candidate is low-threshold voltage-dependent $\mathrm{K}^{+}$channel (e.g., $\mathrm{K}_{\mathrm{v}} 1.2$ ), the expression of which in rodents increases strongly during postnatal development (Wang et al., 1998; Dodson et al., 2002). A developmental increase in low-threshold voltagedependent $\mathrm{K}^{+}$channels would explain the transition of firing pattern from tonic to phasic and a shorter AP width in term MNTB neurons. Other possibilities include a compensating increase in a depolarizing conductance, such as a persistent $\mathrm{Na}^{+}$ current (Huang and Trussell, 2008, Kim et al., 2010). A large sag-depolarization during hyperpolarizing current injections in preterm MNTB neurons might be generated by the activation of hyperpolarizing-activated $\left(\mathrm{I}_{\mathrm{h}}\right)$ channels. The combination of low expression of Kv1.2 and the presence of $\mathrm{I}_{\mathrm{h}}$ may contribute to generate AP bursts during depolarization and a rebound AP after depolarization in preterm MNTB neurons. In term MNTB neurons, an increase of functional Kv1.2 channels might mask the role of $I_{h}$ in regulating the excitability.

A limitation to this study is the influence of environmental factors during postnatal 2 weeks in preterm baboons. Medications, hormones, and nutrients were administered to maintain viability and support normal development, similar to standard care of preterm human infants. It remains to be determined whether these changes are due only to gestational differences or whether standard postnatal care causes abnormal development.

\section{References}

Awatramani GB, Turecek R, Trussell LO (2005) Staggered development of GABAergic and glycinergic transmission in the MNTB. J Neurophysiol 93:819-828. CrossRef Medline
Banks MI, Pearce RA, Smith PH (1993) Hyperpolarization-activated cation current (Ih) in neurons of the medial nucleus of the trapezoid body. J Neurophysiol 70:1420-1432. Medline

Bazwinsky I, Bidmon HJ, Zilles K, Hilbig H (2005) Characterization of the rhesus monkey superior olivary complex by calcium binding proteins and synaptophysin. J Anat 207:745-761. CrossRef Medline

Blanco CL, McGill-Vargas LL, McCurnin D, Quinn AR (2013) Hyperglycemia increases the risk of death in extremely preterm baboons. Pediatr Res 73:337-343. CrossRef Medline

Cant NB (1998) Structural development of the mammalian auditory pathways. In: Springer handbook of auditory research, Vol 9, pp 315-414. New York: Springer.

Dodson PD, Barker MC, Forsythe ID (2002) Two heteromeric Kv1 potassium channels differentially regulate action potential firing. J Neurosci 22:6953-6961. Medline

Downie AL, Jakobson LS, Frisk V, Ushycky I (2002) Auditory temporal processing deficits in children with periventricular brain injury. Brain Lang 80:208-225. CrossRef Medline

Edwards DA, Henderson-Smart DJ, Pettigrew AG, Wetzlar A, Phippard AF (1994) Brainstem auditory evoked response development in preterm and term baboons (Papio hamadryas). Brain Res Dev Brain Res 82:181-184. CrossRef Medline

Grothe B, Pecka M, McAlpine D (2010) Mechanisms of sound localization in mammals. Physiol Rev 90:983-1012. CrossRef Medline

Hall JW 3rd (2000) Development of the ear and hearing. J Perinatol 20:S12S20. CrossRef Medline

Hoffpauir BK, Kolson DR, Mathers PH, Spirou GA (2010) Maturation of synaptic partners: functional phenotype and synaptic organization tuned in synchrony. J Physiol 588:4365-4385. CrossRef Medline

Holcomb PS, Hoffpauir BK, Hoyson MC, Jackson DR, Deerinck TJ, Marrs GS, Dehoff M, Wu J, Ellisman MH, Spirou GA (2013) Synaptic inputs compete during rapid formation of the calyx of Held: a new model system for neural development. J Neurosci 33:12954-12969. CrossRef Medline

Huang H, Trussell LO (2008) Control of presynaptic function by a persistent $\mathrm{Na}(+)$ current. Neuron 60:975-979. CrossRef Medline

Iliadou V, Bamiou DE, Kaprinis S, Kandylis D, Vlaikidis N, Apalla K, Psifidis A, Psillas G, St Kaprinis G (2008) Auditory processing disorder and brain pathology in a preterm child with learning disabilities. J Am Acad Audiol 19:557-563. CrossRef Medline

Kapfer C, Seidl AH, Schweizer H, Grothe B (2002) Experience-dependent refinement of inhibitory inputs to auditory coincidence-detector neurons. Nat Neurosci 5:247-253. CrossRef Medline

Kim JH, Kushmerick C, von Gersdorff H (2010) Presynaptic resurgent $\mathrm{Na}^{+}$ currents sculpt the action potential waveform and increase firing reliability at a CNS nerve terminal. J Neurosci 30:15479-15490. CrossRef Medline

Koch U, Braun M, Kapfer C, Grothe B (2004) Distribution of HCN1 and HCN2 in rat auditory brainstem nuclei. Eur J Neurosci 20:79-91. CrossRef Medline

Moore DR, Jeffery G (1994) Development of auditory and visual systems in the fetus. In: Textbook of fetal physiology, pp 278-286. Oxford: Oxford UP.

Moore JK, Linthicum FH Jr (2007) The human auditory system: a timeline of development. Int J Audiol 46:460-478. CrossRef Medline

Querleu D, Renard X, Boutteville C, Crepin G (1989) Hearing by the human fetus? Semin Perinatol 13:409-420. Medline

Rusu SI, Borst JG (2011) Developmental changes in intrinsic excitability of principal neurons in the rat medial nucleus of the trapezoid body. Dev Neurobiol 71:284-295. CrossRef Medline

Sonntag M, Englitz B, Typlt M, Rübsamen R (2011) The calyx of held develops adult-like dynamics and reliability by hearing onset in the mouse in vivo. J Neurosci 31:6699-6709. CrossRef Medline

Turecek R, Trussell LO (2002) Reciprocal developmental regulation of presynaptic ionotropic receptors. Proc Natl Acad Sci U S A 99:13884-13889. CrossRef Medline

Vles JSH, Casaer P, Kingma H, Swennen C, Daniels HA (1987) Longitudinal study of brainstem auditory evoked potentials of preterm infants. Dev Med Child Neurol 29:577-585.

Wang LY, Gan L, Forsythe ID, Kaczmarek LK (1998) Contribution of the Kv3.1 potassium channel to high-frequency firing in mouse auditory neurones. J Physiol 509:183-194. CrossRef Medline

Wible B, Nicol T, Kraus N (2005) Correlation between brainstem and cortical auditory processes in normal and language-impaired children. Brain 128:417-423. CrossRef Medline 